Title: Liver zonation in children with NAFLD: associations with dietary fructose and uric acid concentrations

\title{
Authors:
}

Nobili Valerio ${ }^{1,2}$, MD; nobili66@yahoo.it ; Mosca Antonella ${ }^{1 *}, M D ;$ antonella.mosca@opbg.net ; Rita De Vito ${ }^{3}, M D$; rita.devito@opbg.net ; Raponi Massimiliano ${ }^{4}, M D$; massimiliano.raponi@opbg.net ; Scorletti Eleonora ${ }^{5}, M D$; e.scorletti@soton.ac.uk; Byrne Christopher $D^{5,6}, M D ;$ c.d.byrne@soton.ac.uk

1.Hepatometabolic Unit - Bambino Gesù Children's Hospital, Rome, Italy

2. Department of Pediatrics, Facoltà di Medicina e Psicologia, Sapienza University of Rome, Italy

3. Histopathology Unit, Bambino Gesù Hospital, IRCCS, Rome, Italy

4. Medical Directorate, "Bambino Gesù" Children's Hospital, IRCCS (Istituto di Ricovero e Cura a Carattere Scientifico), Rome, Italy

5. Human Development and Health Academic Unit, Faculty of Medicine, University of Southampton, Southampton, UK

6. NIHR Southampton Biomedical Research Centre, University Hospital Southampton NHS Foundation Trust and University of Southampton, Southampton, United Kingdom

\section{*Co-first authors}

Correspondence to: Valerio Nobili, MD,

Head of Hepato-Metabolic Disease Unit, “Bambino Gesù” Children’s Hospital IRCCS

(Istituto di Ricovero e Cura a Carattere Scientifico), P.le S. Onofrio, 4 - 00165, Rome, Italy, Phone/Fax: +39-06-68592192, e-mail: valerio.nobili@opbg.net

Short Title: Liver zonation in children with NAFLD

An electronic word count for main body of manuscript $=3359$

Number of figures and tables: 5 tables plus 1 Supplementary Table

References: 36 


\section{List of Abbreviations:}

NAFLD $=$ Non-alcoholic fatty liver disease; $\mathbf{N A F L}=$ Non alcoholic fatty liver; $\mathbf{N A S H}=$ Non-alcoholic steatohepatitis; MetS=metabolic Syndrome; $\mathbf{I R}=$ insulin resistance,

T2DM=type 2 diabetes; $\mathbf{U A}=$ uric acid; $\mathbf{A T P}=$ adenosine triphosphate, $\quad \mathbf{A M P}=$ adenosine monophosphate; IMP= inosine monophosphate; $\quad \mathbf{B M I}=$ body mass index; $\quad \mathbf{L D L}=$ low-density lipoprotein; $\mathbf{A S T}=$ aspartate-aminotransferase; $\mathbf{A L T}=$ alanine-aminotransferases; $\mathbf{G G T}=$ gammaglutamyl-transpeptidase; INR=International Normalized Ratio; OGTT= Oral Glucose Tolerance Test $; \mathbf{W H O}=$ World Health Organization; PNFI $=$ Pediatric NAFLD Fibrosis Index; $\quad$ NAS= NAFLD activity score $; \mathbf{C R N}=\mathrm{NASH}$ Clinical Research Network $; \mathbf{F C D B s}=$ food composition database; INRAN=National Italian Institute of Food Research and Nutrition ; CREA=Centro ricerca per gli alimenti e la nutrizione; SINU=Italian Society of Human Nutrition.

Conflict of interest: All authors declare that there is no conflict of interest that could be perceived as affecting the impartiality of the reported research.

A Statement of Financial support: No external funding supported the research described in this manuscript. This research did not receive any specific grant from any funding agency in the public, commercial or not-for-profit sector.

Authors contribution: Profs Byrne and Nobili conceptualized and designed the study. All authors approved the final manuscript as submitted and agree to be accountable for all aspects of the work. 


\begin{abstract}
Background\&Aims As dietary components are delivered directly to the periportal zone of the liver lobule, there is the potential for greater injury in this zone (zone1) compared to the perivenous zone (zone3). We determined whether there was differential zonal injury in NAFLD and investigated associations between dietary fructose consumption and uric acid concentrations in periportal and perivenous zones.
\end{abstract}

Methods. 271 children's histological images were scored in five periportal and perivenous zones for steatosis, ballooning, inflammation, and fibrosis severity. Means \pm SDs zonal scores were calculated, for each histological feature. Dietary fructose consumption (grammes/day) was assessed by food frequency questionnaire, and uric acid measured in serum. Logistic regression was undertaken to test associations between both high fructose consumption and hyperuricaemia, and histological disease in periportal and perivenous zones. OR(95\%CIs) are reported.

Results. Mean age 12.5 years. Inflammation was increased in the periportal versus perivenous zones $(0.78 \pm 0.43$ v. $0.41 \pm 0.48, \mathrm{p}=0.041)$. There were non significant trends towards greater steatosis, ballooning and fibrosis in the periportal zone. In the fully adjusted models, high fructose intake $(>43.6 \mathrm{~g} /$ day) was associated with disease in both zones. E.g. for periportal and perivenous zones respectively, steatosis $1.56(1.12,2.49)$ and 1.21(1.09,2.73); inflammation 4.29(2.31,5.88) and 3.69(2.14,4.56); and fibrosis 2.72(1.43,3.76) and 1.96(1.24,2.37). Hyperuricaemia was associated with inflammation only in the periportal zone 1.71(1.17,2.35); and was associated with steatosis and fibrosis in both zones; e.g. for periportal and perivenous zones respectively, steatosis 2.98(1.65,3.23) and 1.14(1.05,1.99); and fibrosis, 2.65(1.35,2.99) and 1.31(1.13,2.17).

Conclusions. High fructose consumption is associated with disease severity in both lobular zones and hyperuricaemia may be associated with more severe disease in the periportal zone.

Keywords: Liver zonation, Periportal, perivenous, lobule, liver cell plate, uric acid, dietary fructose, Non-alcoholic fatty liver disease (NAFLD), non alcoholic steatohepatitis (NASH) 


\section{Key points box}

- Recently, it is demonstrated that serum UA concentration and fructose consumption are associated with NASH.

- In children with NAFLD, there were greater levels of inflammation in the periportal compared to perivenous zones.

- We show significant associations between high dietary fructose consumption and steatosis, inflammation and fibrosis, in both the periportal and perivenous zones

- Hyperuricaemia was associated with steatosis and fibrosis in both the periportal and perivenous zones. 


\section{Introduction}

Within the liver, hepatocytes are arranged along the lobule which is a unique architectural anatomical structure. The lobule is made up of cells arranged as liver plates and the cells (e.g. hepatocytes, Kupffer cells and stellate cells) are arranged along the liver plate bordering hepatic sinusoids and biliary ductules. Each plate comprises $15-25$ cells bordering the sinusoid, and at either end of the sinusoids, there are distinct regions referred to as periportal (zone 1) and perivenous zones (zone 3)(1).

According to their position across the liver cell plate, hepatocytes are exposed to different concentrations of dietary nutrients and oxygen tensions(1-3). In the periportal zone, the portal venules deliver venous blood rich in dietary nutrients from the intestine. In this zone, portal venous blood mixes with arterial blood from the hepatic artery and because of the presence of this arterial blood, periportal hepatocytes are exposed to a high oxygen tension. Mixed portal venous blood and arterial blood flows along the sinusoid to the perivenous zone and then drains into the central veins, before leaving the liver, via the hepatic veins. Thus, hepatocytes have specialized metabolic functions according to their specific location along the hepatic sinusoid. This zonation of hepatic functions mainly affects glucose/energy metabolism, ammonia detoxification and xenobiotic metabolism(1). With regard to glucose/energy metabolism, hepatocytes located in the periportal zone specialize in functions such as beta oxidation that require high oxygen tension, whereas in contrast, perivenous hepatocytes specialize in functions such as lipogenesis, and glycolysis, that can occur at much lower oxygen concentrations $(1,4-7)$.

Consequently, differential hepatocyte exposure to dietary nutrients (and toxins), and oxygen tension, together with specialization of metabolic functions according to position in the liver cell plate, creates the potential for zonal-specific liver injury, particularly as a consequence of harmful dietary stimuli. Recently, evidence from the study of liver tissue obtained in mouse models of NAFLD, and patients with non alcoholic fatty liver (NAFL) or non alcoholic steatohepatitis 
(NASH), has shown that there are several lipid species that have distinct zonal distributions, particularly in NAFL (8). Moreover, this study revealed that there is also partial to complete loss of lipid zonation when the liver disease has progressed to NASH. This work supports the results of a previous study undertaken by other investigators who had shown that there is zonation of specific choline-containing phospholipids in human liver, that is also either lost, or preserved, according to NAFLD severity(9). Thus, NAFLD is associated not only with lipid enrichment, but also with zonal changes of specific lipids. Thus differential changes in lipid species, and their associated metabolic pathways, may play a role in the heterogeneous progression of liver disease in NAFLD.

Recently, in 271 children with proven NAFLD, we tested whether dietary fructose consumption was associated with $\mathrm{NASH}(10)$. We showed that fructose consumption was independently associated with NASH and because high fructose consumption is correlated with high serum uric acid levels, we also investigated the association between uric acid concentrations and NASH. In that additional analysis, we showed that hyperuricaemia was also independently and positively associated with NASH(10). As a result of our work, Mann and Armstrong queried whether we had observed specific patterns of zonal liver injury associated with fructose intake (11), which at that time we had not studied(12).

As a direct consequence of the correspondence and our original study (10), supported by further evidence that zonal-specific lipid changes may occur in $\operatorname{NAFL}(8,9)$, we reasoned that in children with NAFLD, there would be differences in liver disease severity between periportal and perivenous zones. Additionally, assuming that differences in liver disease severity exist between periportal and perivenous zones, since hepatocytes in the periportal zone would be exposed to higher concentrations of fructose (via the portal blood supply to this zone), we hypothesized (supported by a review of the subject(13)), that high dietary fructose consumption would be associated with increased severity of liver disease specifically in the periportal zone. Our aim was 
therefore to determine whether there were zonal differences in the histological severity of liver disease between the periportal and perivenous zones of the liver lobule based on the original descriptions of zonation of liver parenchyma proposed by Jungermann (2-4). In addition, we analysed associations between: i) dietary fructose consumption and ii) uric acid concentrations, and histological disease severity, in both the periportal (zone 1) and the perivenous zones (zone 3). 


\section{PATIENTS AND METHODS}

\section{Anthropometrical and biochemical measurements}

Overweight/obese children who were referred to the "Hepatometabolic Department" of the “Bambino Gesù” Children's Hospital, from January 2012 to November 2014, underwent liver biopsy for suspected NASH. 271 consecutive children were enrolled in this study and all parents gave written informed consent. During the initial investigations, liver fat was identified by ultrasonography using established criteria, a bright hepatic echo pattern compared to echo response of the right kidney (14). Other causes of steatosis, were excluded, such as total parenteral nutrition, use of drugs known to induce steatosis (e.g. valproate, amiodarone or prednisone), viral hepatitis (A, B, C, Cytomegalovirus and Epstein-Barr virus), autoimmune or metabolic liver diseases, alpha1-antitrypsin deficiency, Wilson's disease, and celiac disease. Patients with diabetes, genetic dyslipidaemia and genetic syndromes were excluded.

Anthropometric and clinical parameters (weight, height, body mass index- BMI, waist circumference-WC) were measured in all patients using standardized methods. Total cholesterol, LDL-cholesterol, Triglycerides, uric acid (UA) and liver function tests (LFT's - aspartate- (AST) and alanine- (ALT) aminotransferases, gamma-glutamyl-transpeptidase (GGT), bilirubin, albumin and International Normalized Ratio (INR)) were measured by standard methods. Moreover, in all patients an Oral Glucose Tolerance Test (OGTT) was performed according to the recommendations of the World Health Organization (WHO)(15).

\section{Liver biopsy}

According to the recommendation of the Hepatology Committee of the European Society of Paediatric Gastroenterology, Hepatology and Nutrition (ESPGHAN), all patients underwent liver biopsy in order to exclude other liver diseases, or to assess the severity of liver disease, suspected by clinical and laboratory evaluation using tests such as hypertransaminasemia, hepatosplenomegaly, or the presence of a high Paediatric NAFLD Fibrosis Index (PNFI)(16, 17). 
Liver biopsies were performed in all children using an automatic core biopsy device (Biopince, Amedic, Sweden) with an $18-\mathrm{G}$ needle that is $150 \mathrm{~mm}$ long (18). All biopsies were at least $18 \mathrm{~mm}$ in length and were assessed by a single liver histo-pathologist who was unaware of the patient's clinical and laboratory data. Biopsies were routinely processed (formalin-fixed, paraffin-embedded) and analysed by different staining. A Zeiss AXIO Imager microscope with a Zeiss digital camera was used for image capturing. For each child's biopsy, 10 fields from sections stained with E\&E and ten fields from sections stained with Masson Thricrome were photographed at objective x20, and stored in a computer using AXIO CamMRc5 software. For both stains, five fields were counted in the periportal zone (zone 1) and five fields were counted in the perivenous zone (zone 3). All images were evaluated for steatosis severity ( $0-3$ points), ballooning severity ( $0-2$ points), and inflammation severity (0-2 points), according to the Scoring System for Non-Alcoholic Fatty Liver Disease developed by the NIH-sponsored NASH Clinical Research Network (CRN)(19). With regard to the assessment of fibrosis severity; the NASH CRN system scores portal and periportal fibrosis as absent or present (i.e. stage 1C). Centrilobular fibrosis is scored as absent or present and as mild (stage 1A) or moderate (stage 1B). Previously, we have reported in Italian children with NAFLD that $87 \%$ of children with NAFLD, presented with Stage $1 \mathrm{C}$ and only $2.5 \%$ and $10 \%$ presented stage $1 \mathrm{~A}$ and $1 \mathrm{~B}$ respectively(20). Therefore, when fibrosis occurs in children with NAFLD it is usually mild, and because our intention was to compare zones 1 and 3, we adopted a dichotomous scoring system for assessing fibrosis in both zones, scoring the absence of fibrosis as $=0$, and the presence of fibrosis as $=1$. For each assessed histological parameter (steatosis, ballooning, inflammation and fibrosis, the mean value of five fields was calculated in both the periportal and perivenous zones. For the whole cohort, $n=271$, a mean $\pm \mathrm{SD}$ value was calculated for presentation of the characteristics of the cohort in both zones. For each histological characteristic, we tested whether the distributions for steatosis, ballooning, inflammation, fibrosis and NAS score, were normally distributed in both zones. 
The mean value for each histological parameter in either zone in each subject was used in correlation and logistic regression analyses, as shown in the Results section. The presence of NASH within the whole sample was defined according to the NAS (a NAS of 5 or greater defined the presence of NASH). Because the inflammation score in NAS specifically refers to lobular inflammation, we did not determine a NAS for periportal versus perivenous zones.

\section{Assessment of dietary fructose consumption}

A food frequency questionnaire (FFQ) was completed by all patients as previously described(21). The frequency intake of a particular food was defined as follows: 'every day of the week', 'sometimes', and 'never'. The questionnaire included breakfast, morning snack, lunch, afternoon snack, and dinner, and examined the intake of specific foods and portions. The food composition database (FCDBs), shown in the book of LARN - IV Edition, published by the National Italian Institute of Food Research and Nutrition (INRAN-CREA) and UK charts was used to assess the intake of fructose per day (tables only contain data, where the content of free fructose is known) (22). The study design conformed to the ethical guidelines of the Declaration of Helsinki (as revised in Seoul, Korea, October 2008) and was approved by the local Ethics Committee for Bambino Gesù” Children’s Hospital (ID Prot. 323/12).

\section{Statistical analysis}

Statistical analyses were undertaken using STATISTICA software (version 2010, Chicago, IL, USA). The Kolmogorov-Smirnov test was used to determine whether variables were normally distributed. Normally distributed data are described as means \pm standard deviations (SDs) and nonnormally distributed data are expressed as medians and IQRs. The study population was divided into quartiles based on daily intake of dietary fructose. Between group differences were analysed by Kruskal-Wallis tests (for non normally distributed data) or analysis of variance (ANOVA). Pearson's and Spearman's correlation tests were used to test univariate associations between 
exposures and outcomes. Binary logistic regression was used to test associations between: a) high fructose consumption $\left(1=>43.6 \mathrm{gr} /\right.$ day, $0=\leq 43.6 \mathrm{gr} /$ day, equal to $50^{\text {th }}$ percentile of fructose concentration in our population) and b) hyperiuricaemia ( $\geq 5.9 \mathrm{mg} / \mathrm{dL})$ as the key outcomes, with the histological parameters of interest, as the exposures. Other covariates and potential confounders were also entered as exposures into the regression models. A p value of $<0.05$ was considered statistically significant. Logistic regression analyses were undertaken using SPSS software (IBM SPSS Statistics for Windows, version 20.0, Armonk, NY). 


\section{Results}

271 consecutive children (155 males) with NAFLD, mean age 12.5 years, were included in the study. $37.6 \%(n=102)$ of patients had NASH and $62.4 \%(n=169)$ did not have NASH (Not-NASH) defined by the NAS. Patients were stratified into four groups by quartiles of dietary fructose intake. Mean \pm SD dietary fructose intake (grammes/day) in these groups were: quartile 1, 21.53 \pm 1.99 ; quartile 2, 31.64 \pm 2.76 ; quartile $3,47.26 \pm 9.81$; and quartile $4,88.39 \pm 16.25$.

Table 1 shows the between group differences in anthropometric and biochemical characteristics according to fructose consumption. Patients in the highest quartile of dietary fructose consumption had the highest BMI, ALT, serum triglyceride and UA concentrations and HOMA-IR.

Table 2 shows means \pm SDs for five field in both periportal and perivenous lobular zones for steatosis, inflammation, ballooning and fibrosis. Inflammation was greater in the periportal zone.

Table 3 describes the histological characteristics in periportal and perivenous zones according to quartiles of dietary fructose intake. These data suggested differences for steatosis, inflammation and fibrosis between periportal and perivenous zones in subjects within the highest quartile of dietary fructose intake. Additionally, for the periportal zone, there were significant between group differences, for steatosis, inflammation and fibrosis $(\mathrm{p}<0.05)$.

Table 4 describes the histological characteristics in periportal and perivenous zones according to serum uric acid concentrations above and below the threshold used to define hyperuricaemia (uric acid $<5.9$ and uric acid $\geq 5.9(23))$. These data showed that there were no significant differences by zone in levels of steatosis. In contrast levels of fibrosis were higher in periportal versus perivenous zones, above and below the threshold of uric acid concentration used to define hyperuricaemia. In contrast, levels of inflammation were higher in the periportal zone, only in patients with hyperuricaemia. 
We also studied the associations between dietary fructose intake (grammes/day) and serum uric acid concentration $(\mathrm{mg} / \mathrm{dL})$ as continuous variables, and histological parameters (Supplementary Table 1). These data showed there were significant correlations between dietary fructose intake and both steatosis and fibrosis in periportal and perivenous zones, and there was a significant correlation between fructose intake and inflammation only in the periportal zone. For uric acid, there were significant correlations between serum uric acid concentration and steatosis, inflammation and fibrosis, only in the periportal zone.

Next we tested whether high dietary fructose consumption or hyperuricaemia (serum uric acid concentration $\geq 5.9 \mathrm{mg} / \mathrm{dL}$ ) were independently associated with histological parameters (steatosis, inflammation, ballooning, or fibrosis), adjusting the regression models for potential confounders. Since BMI, HOMA-IR, and serum triglyceride and uric acid concentrations were higher in subjects in the highest quartile of dietary fructose intake, when testing the independence of associations with high dietary fructose intake, we adjusted the regression models for these potential confounders (Table 5). These data showed that in the fully adjusted model, high fructose consumption was associated with histological parameters (steatosis, inflammation and fibrosis) in both periportal and perivenous zones. When testing the independence of associations between histological parameters and hyperuricaemia, the regression models were adjusted for BMI, HOMA-IR, serum triglyceride and dietary fructose intake (Table 5). These data showed significant associations between hyperuricaemia and steatosis in periportal and perivenous zones; inflammation in the periportal zone only, and fibrosis in both periportal and perivenous zones. 


\section{DISCUSSION}

Our results show that in children with NAFLD, there were significantly greater levels of inflammation in periportal compared to perivenous zones, and there was also a non significant trend toward greater steatosis, ballooning and fibrosis in the periportal compared to periportal zones. We also show significant associations between high dietary fructose consumption and steatosis, inflammation and fibrosis, in both the periportal and perivenous zones. Hyperuricaemia was associated with steatosis and fibrosis in both the periportal and perivenous zones, whereas liver inflammation was associated with hyperuricaemia only in the periportal zone. Increasing evidence suggests that pediatric NAFLD is different from adult NAFLD and may involve greater periportal liver injury(24-26). Taken together with our results, the data highlight that increased dietary fructose consumption coupled with hyperuricaemia, could cause greater damage in the periportal zone rather than the perivenous zone. Thus, we speculate that high dietary fructose intake and hyperuricemia, could at least in part, be responsible for the different pattern of lobular disease observed in pediatric NAFLD.

Since, portal venules deliver dietary nutrients (such as fructose) directly to the periportal zone and hepatocytes in this region are exposed to higher oxygen tension and these hepatocytes specialize in oxidative metabolism, we postulated that high dietary fructose intake would be more strongly associated with liver injury in the periportal zone (zone 1). That we found associations between high dietary fructose intake and liver injury in the perivenous lobular zone, suggests that there is no lobular sparing and high dietary fructose intake is also potentially harmful to hepatocytes in this zone of the liver plate. High dietary fructose consumption is associated with increased uric acid concentrations; and experimental data demonstrates oxidative stress is one of the earliest phenomena observed when liver cells are exposed to uric acid(27-33). Since oxygen tension is highest in the periportal zone, we investigated whether hyperuricaemia was associated with differential patterns of zonal injury, hypothesizing that zonal injury linked to oxidative stress might 
be greater in the perportal zone. There was a suggestion in our data that zonal injury associated with hyperuricaemia was greater in the periportal zone, as inflammation was only associated with hyperuricaemia in this zone.

Fructose is a monosaccaride contained within sugar (sucrose), high fructose corn syrup, honey and fruits. After absorption from the diet, fructose is transported in the portal circulation to the liver where it enters glycolysis and is phosphorylated to fructose-1-phosphate. The phosphorylation of fructose depletes cellular adenosine triphosphate and stimulates adenosine monophosphate (AMP) deaminase, to convert AMP to inosine monophosphate (IMP) and then IMP is converted to uric acid(27). In children soft drinks, rather than fruit, can provide substantial quantities of dietary fructose. It has been suggested that the marked increase in fructose intake may have a role in the rising levels of serum uric acid and obesity worldwide $(34,35)$. It has been shown that uric acidinduced oxidative stress and steatosis in liver cells in culture, and attenuation of this phenomenon by anti-oxidants suggests that both NADPH-oxidase dependent and mitochondrial generation oxidative stress are responsible for uric acid-mediated hepatic fat accumulation $(29,36)$. Our data shows that both high dietary fructose consumption and hyperuricaemia were associated with steatosis in both the periportal and perivenous zones. These data, and the plausible biological mechanisms linking fructose, uric acid and liver fat accumulation suggest there is an extensive cellular impact of high dietary fructose intake within the cells of the liver plate. We cannot be certain with the nature of our study that all liver cell plates are equally affected throughout the liver, but for each liver zone, five different liver plates were examined and there was very little variation between liver cell plates in each of the 271 patients' livers that were examined.

There are strengths and limitations of our study that should be considered. We have studied 271 children and adolescents who have undergone liver biopsy to assess the histological severity of NAFLD. We have assessed the severity of disease for steatosis, ballooning, inflammation and 
fibrosis in each child's liver biopsy, in five fields, in both the periportal and perivenous zones. We have calculated means \pm SD for steatosis, ballooning, inflammation and fibrosis scores in both periportal and perivenous zones for each child. For each histological characteristic we tested whether the distributions for steatosis, ballooning, inflammation and fibrosis scores were normally distributed. All subjects completed a dietary questionnaire to assess their fructose consumption but it is possible that this assessment may not truly reflect all dietary consumption of fructose. However, any misclassification bias would tend to attenuate the strength of our findings, and would bias our results towards the null. We are not able to prove that increased concentrations of UA are solely due to high dietary fructose intake and it is possible that high levels of purine containing foods contributed to increased UA concentrations. Additionally another limitation is that it was not possible to have a control group of children with proven NAFLD and no dietary intake of fructose, in order to compare zonal patterns of disease in such a group.

In conclusion, in a cohort of children with a histological diagnosis of NAFLD, we show there were greater levels of inflammation in periportal compared to perivenous zones. We also show significant independent associations between high dietary fructose consumption and steatosis, inflammation and fibrosis, in both the periportal and perivenous zones. Hyperuricaemia was independently associated with both steatosis and fibrosis in both the periportal and perivenous zones, whereas liver inflammation was independently associated with hyperuricaemia in only the periportal zone.

\section{Acknowledgements}

ES and CDB are supported in part by the Southampton NIHR Biomedical Research Centre. 



\section{REFERENCES}

1. COLNOT S P, C. Liver zonation. In: Monga S P S, ed. Molecular Pathology of Liver Diseases: Springer Science+Business Media, 2011: 7-16.

2. JUNGERMANN K, KIETZMANN T. Zonation of parenchymal and nonparenchymal metabolism in liver. Annual review of nutrition 1996; 16: 179-203.

3. JUNGERMANN K. Metabolic zonation of liver parenchyma. Seminars in liver disease 1988; 8(4): 329-41.

4. JUNGERMANN K. Role of intralobular compartmentation in hepatic metabolism. Diabete \& metabolisme 1992; 18(1 Pt 2): 81-6.

5. BURNS S P, COHEN R D, ILES R A, et al. Zonation of gluconeogenesis, ketogenesis and intracellular $\mathrm{pH}$ in livers from normal and diabetic ketoacidotic rats: evidence for intralobular redistribution of metabolic events in ketoacidosis. The Biochemical journal 1999; $343 \mathrm{Pt} 1: 273-80$. 6. BURNS S P, MURPHY H C, ILES R A, BAILEY R A, COHEN R D. Hepatic intralobular mapping of fructose metabolism in the rat liver. The Biochemical journal 2000; 349(Pt 2): 539-45. 7. BRASS C A, CRAWFORD J M, NARCISO J, GOLLAN J L. Hypoxic liver injury and the ameliorating effects of fructose: the "glucose paradox" revisited. Am J Physiol 1992; 263(3 Pt 1): G293-300.

8. HALL Z, BOND N J, ASHMORE T, et al. Lipid zonation and phospholipid remodeling in nonalcoholic fatty liver disease. Hepatology 2017; 65(4): 1165-80.

9. WATTACHERIL J, SEELEY E H, ANGEL P, et al. Differential intrahepatic phospholipid zonation in simple steatosis and nonalcoholic steatohepatitis. PLoS One 2013; 8(2): e57165.

10. MOSCA A, NOBILI V, DE VITO R, et al. Serum uric acid concentrations and fructose consumption are independently associated with NASH in children and adolescents. J Hepatol 2017; 66(5): 1031-36.

11. MANN J P, ARMSTRONG M J. Fructose, uric acid, and zonal differences in NASH. J Hepatol 2017.

12. NOBILI V, MOSCA A, SCORLETTI E, BYRNE C D. Reply to: "Fructose, uric acid and zonal differences in NASH". J Hepatol 2017.

13. HIJMANS B S, GREFHORST A, OOSTERVEER M H, GROEN A K. Zonation of glucose and fatty acid metabolism in the liver: mechanism and metabolic consequences. Biochimie 2014; 96: 121-9.

14. WANG C C, TSENG T C, HSIEH T C, et al. Severity of fatty liver on ultrasound correlates with metabolic and cardiovascular risk. The Kaohsiung journal of medical sciences 2012; 28(3): 151-60.

15. WORLD HEALTH ORGANIZATION (WHO) I D F I. Definition and diagnosis of diabetes mellitus and intermediate hyperglycemia. Report of a WHO/IDF Consultation 2006.

16. DEZSOFI A, BAUMANN U, DHAWAN A, et al. Liver biopsy in children: position paper of the ESPGHAN Hepatology Committee. Journal of pediatric gastroenterology and nutrition 2015; 60(3): 408-20.

17. NOBILI V, ALISI A, VANIA A, TIRIBELLI C, PIETROBATTISTA A, BEDOGNI G. The pediatric NAFLD fibrosis index: a predictor of liver fibrosis in children with non-alcoholic fatty liver disease. BMC Med 2009; 7: 21.

18. PIETROBATTISTA A, FRUWIRTH R, NATALI G, MONTI L, DEVITO R, NOBILI V. Is juvenile liver biopsy unsafe? Putting an end to a common misapprehension. Pediatric radiology 2009; 39(9): 959-61.

19. BRUNT E M, KLEINER D E, WILSON L A, BELT P, NEUSCHWANDER-TETRI B A. Nonalcoholic fatty liver disease (NAFLD) activity score and the histopathologic diagnosis in NAFLD: distinct clinicopathologic meanings. Hepatology 2011; 53(3): 810-20. 
20. NOBILI V, MANCO M, DEVITO R, et al. Lifestyle intervention and antioxidant therapy in children with nonalcoholic fatty liver disease: a randomized, controlled trial. Hepatology 2008; 48(1): 119-28.

21. NOBILI V, LICCARDO D, BEDOGNI G, et al. Influence of dietary pattern, physical activity, and I148M PNPLA3 on steatosis severity in at-risk adolescents. Genes \& nutrition 2014; 9(3): 392.

22. Food Composition Table.

23. CHO S M, LEE S G, KIM H S, KIM J H. Establishing pediatric reference intervals for 13 biochemical analytes derived from normal subjects in a pediatric endocrinology clinic in Korea. Clinical biochemistry 2014; 47(18): 268-71.

24. SCHWIMMER J B, BEHLING C, NEWBURY R, et al. Histopathology of pediatric nonalcoholic fatty liver disease. Hepatology 2005; 42(3): 641-9.

25. TAKAHASHI Y, FUKUSATO T. Pediatric nonalcoholic fatty liver disease: overview with emphasis on histology. World J Gastroenterol 2010; 16(42): 5280-5.

26. FLEET S E, LEFKOWITCH J H, LAVINE J E. Current Concepts in Pediatric Nonalcoholic Fatty Liver Disease. Gastroenterology clinics of North America 2017; 46(2): 217-31.

27. KANG D H, HA S K. Uric Acid Puzzle: Dual Role as Anti-oxidantand Pro-oxidant. Electrolyte Blood Press 2014; 12(1): 1-6.

28. SANCHEZ-LOZADA L G, LANASPA M A, CRISTOBAL-GARCIA M, et al. Uric acidinduced endothelial dysfunction is associated with mitochondrial alterations and decreased intracellular ATP concentrations. Nephron Experimental nephrology 2012; 121(3-4): e71-8. 29. LANASPA M A, SANCHEZ-LOZADA L G, CHOI Y J, et al. Uric acid induces hepatic steatosis by generation of mitochondrial oxidative stress: potential role in fructose-dependent and independent fatty liver. J Biol Chem 2012; 287(48): 40732-44.

30. RYU E S, KIM M J, SHIN H S, et al. Uric acid-induced phenotypic transition of renal tubular cells as a novel mechanism of chronic kidney disease. American journal of physiology Renal physiology 2013; 304(5): F471-80.

31. SAUTIN Y Y, NAKAGAWA T, ZHARIKOV S, JOHNSON R J. Adverse effects of the classic antioxidant uric acid in adipocytes: NADPH oxidase-mediated oxidative/nitrosative stress. Am J Physiol Cell Physiol 2007; 293(2): C584-96.

32. YU M A, SANCHEZ-LOZADA L G, JOHNSON R J, KANG D H. Oxidative stress with an activation of the renin-angiotensin system in human vascular endothelial cells as a novel mechanism of uric acid-induced endothelial dysfunction. J Hypertens 2010; 28(6): 1234-42.

33. KANG D H, PARK S K, LEE I K, JOHNSON R J. Uric acid-induced C-reactive protein expression: implication on cell proliferation and nitric oxide production of human vascular cells. Journal of the American Society of Nephrology : JASN 2005; 16(12): 3553-62.

34. JOHNSON R J, SEGAL M S, SAUTIN Y, et al. Potential role of sugar (fructose) in the epidemic of hypertension, obesity and the metabolic syndrome, diabetes, kidney disease, and cardiovascular disease. Am J Clin Nutr 2007; 86(4): 899-906.

35. NAKAGAWA T, HU H, ZHARIKOV S, et al. A causal role for uric acid in fructoseinduced metabolic syndrome. American journal of physiology Renal physiology 2006; 290(3): F625-31.

36. CHOI Y J, SHIN H S, CHOI H S, et al. Uric acid induces fat accumulation via generation of endoplasmic reticulum stress and SREBP-1c activation in hepatocytes. Lab Invest 2014; 94(10): 1114-25. 
Table 1 Group differences in anthropometric and biochemical characteristics according to quartiles of fructose consumption

\begin{tabular}{|c|c|c|c|c|c|}
\hline & $\begin{array}{l}\text { Dietary fructose } \\
\text { intake } \\
\text { Quartile } 1 \\
(\mathrm{n}=\mathbf{3 0})\end{array}$ & \begin{tabular}{|l} 
Dietary fructose \\
intake \\
Quartile 2 \\
$(n=54)$
\end{tabular} & $\begin{array}{l}\text { Dietary fructose } \\
\text { intake } \\
\text { Quartile } 3 \\
(n=110)\end{array}$ & $\begin{array}{l}\text { Dietary fructose intake } \\
\text { Quartile } 4 \\
N=(82)\end{array}$ & $\mathbf{p}$ \\
\hline Age (years) & $11.5(1.33)$ & $11.1(1.83)$ & $10.57(3.25)$ & $10.36(2.56)$ & 0.73 \\
\hline $\operatorname{Sex}(\mathbf{F} / \mathbf{M} \%)$ & $16 / 14$ & $36 / 18$ & $67 / 43$ & $29 / 53$ & \\
\hline Weight ( mean \pm SD ) & $53.55(9.59)$ & $58.11(15.14)$ & $57.75(23.41)$ & $64.37(14.96)$ & 0.002 \\
\hline BMI, Kg/mq ( mean \pm SD) & $25.4(3 . .91)$ & $26.7(4.32)$ & $27.10(5.31)$ & $29.71(4.62)$ & 0.033 \\
\hline WC, cm ( mean \pm SD ) & $84.84(8.54)$ & $86.93(9.35)$ & $83.97(12.77)$ & $93.81(9.93)$ & 0.97 \\
\hline AST, UI/L ( median; IQR) & $41(15.44)$ & $46(16-77)$ & $45(25-66)$ & $46(18-58)$ & 0.89 \\
\hline ALT, UI/L ( median; IQR) & $49(22-63)$ & $66(24-78)$ & $72(37-168)$ & $78(45-88)$ & 0.79 \\
\hline Uric Acid, mg/dl ( median; IQR) & $4.4(1.5-5.3)$ & $4.6(1.4-6.2)$ & $5.5(3-6)$ & $7(5-8)$ & 0.003 \\
\hline Hyperuricemia, (uric acid $>5.9 \mathrm{mg} / \mathrm{dl}$ ) $(\%)$ & $5(33.3 \%)$ & $17(38.6 \%)$ & $59(40.7 \%)$ & $44(53.6 \%)$ & \\
\hline Total Cholesterol, mg/dl ( median; IQR) & $158(107-185)$ & $160(127-203)$ & $165(112-232)$ & $185(120-245)$ & 0.37 \\
\hline LDL Cholesterol, mg/dl ( median; IQR) & $83(63-101)$ & $84(65-112)$ & $91(68-134)$ & $101(87-142)$ & 0.55 \\
\hline HDL cholesterol, mg/dl ( median; IQR) & $56(41-68)$ & $54(37-67)$ & $44(27-58)$ & $38(21-53)$ & 0.13 \\
\hline Triglycerides, mg/dl ( median; IQR) & $99(62-122)$ & $104(65-168)$ & $119(60-125)$ & $154(56-198)$ & 0.019 \\
\hline $\begin{array}{l}\text { Fasting plasma glucose, mg/dl ( median; } \\
\text { IQR) }\end{array}$ & $85(64-96)$ & $84(74-102)$ & $82(74-110)$ & $92(82-106)$ & 0.15 \\
\hline Fasting plasma gluc-120'( median; IQR) & $99(88-113)$ & $111(98-131)$ & $118(82-141)$ & $121(88-161)$ & 0.65 \\
\hline Fasting Insulin, mU/L ( median; IQR) & $13.5(9-21)$ & $14(9-24)$ & $18(6-22)$ & $25(11-38)$ & 0.021 \\
\hline Insulin -120 minute mU/L ( median; IQR) & $91(46-113)$ & $92(36-137)$ & $111(69-135)$ & 132(93-149) & 0.09 \\
\hline HOMA-IR( mean \pm SD ) & $2.89(1.19)$ & $2.98(1.45)$ & $3.13(2.24)$ & $5.16(1.82)$ & 0.035 \\
\hline $\begin{array}{l}\text { Dietary fructose intake } \\
\text { grammes/day (mean } \pm \text { SD) }\end{array}$ & $21.53(1.99)$ & $31.64(2.76)$ & $47.26(9.81)$ & $88.39(16.25)$ & - \\
\hline
\end{tabular}

Kruskal-Wallis tests, $p$ value of $<0.05$

$\mathrm{BMI}=$ body mass index $; \mathrm{WC}=$ waist circumference AST $=$ aspartate aminotransferase $; \mathrm{ALT}=$ alanine aminotransferase $; \mathrm{HOMA}-\mathrm{IR}$

$=$ homeostasis model assessment of insulin resistance 
Table 2. Histological features of NAFLD in periportal and perivenous zones

\begin{tabular}{|l|l|l|l|}
\hline $\mathbf{N}=\mathbf{2 7 1}$ & $\begin{array}{l}\text { Periportal } \\
\text { (zone 1) } \\
\text { Mean } \pm \text { SD }\end{array}$ & $\begin{array}{l}\text { Perivenous } \\
\text { (zone 3) } \\
\text { Mean } \pm \text { SD }\end{array}$ & p value \\
\hline Steatosis & $1.08(0.51)$ & $0.93(0.55)$ & 0.112 \\
\hline Inflammation & $0.78(0.43)$ & $0.41(0.48)$ & $\mathbf{0 . 0 4 1}$ \\
\hline Ballooning & $0.19(0.33)$ & $0.14(0.41)$ & 0.784 \\
\hline Fibrosis & $0.45(0.59)$ & $0.42(0.61)$ & 0.723 \\
\hline
\end{tabular}

Means \pm SDs for five field in both the periportal and perivenous lobular zones, for steatosis, inflammation, ballooning and fibrosis. 
Table 3. Histological features of NAFLD associated with Fructose consumption

\begin{tabular}{|c|c|c|c|c|c|}
\hline & $\begin{array}{l}\text { Dietary fructose intake } \\
\text { Quartile } 1 \\
(\mathrm{n}=30)\end{array}$ & $\begin{array}{l}\text { Dietary fructose intake } \\
\text { Quartile } 2 \\
(n=54)\end{array}$ & $\begin{array}{l}\text { Dietary fructose intake } \\
\text { Quartile } 3 \\
(n=110)\end{array}$ & $\begin{array}{l}\text { Dietary fructose intake } \\
\text { Quartile } 4 \\
(n=82)\end{array}$ & $P^{*}$ \\
\hline Steatosis zone 1 & $0.52 \pm 0.23$ & $0.93 \pm 0.61$ & $1.06 \pm 0.54$ & $1.81 \pm 0.65$ & 0.004 \\
\hline Steatosis zone 3 & $0.42 \pm 0.36$ & $0.92 \pm 0.55$ & $0.96 \pm 0.61$ & $1.03 \pm 0.69$ & 0.101 \\
\hline$p(\mathrm{z} 1$ vs. $\mathrm{z} 3)$ & 0.66 & 0.87 & 0.09 & 0.02 & \\
\hline Inflammation zone 1 & $0.29 \pm 0.31$ & $0.52 \pm 0.55$ & $0.78 \pm 0.54$ & $1.41 \pm 0.23$ & 0.002 \\
\hline Inflammation zone 3 & $0.39 \pm 0.44$ & $0.28 \pm 0.34$ & $0.43 \pm 0.59$ & $0.69 \pm 0.19$ & 0.001 \\
\hline$p(\mathrm{z} 1$ vs. $\mathrm{z} 3)$ & 0.25 & 0.031 & 0.023 & 0.001 & \\
\hline Ballooning zone 1 & $0.11 \pm 0.29$ & $0.10 \pm 0.23$ & $0.20 \pm 0.19$ & $0.44 \pm 0.26$ & 0.033 \\
\hline Ballooning zone 3 & $0.23 \pm 9.18$ & $0.10 \pm 0.21$ & $0.17 \pm 0.14$ & $0.33 \pm 0.11$ & 0.047 \\
\hline$p(\mathrm{z} 1$ vs. $\mathrm{z} 3)$ & 0.46 & 0.94 & 0.86 & 0.85 & \\
\hline Fibrosis zone 1 & $0.22 \pm 0.14$ & $0.39 \pm 0.25$ & $0.50 \pm 0.34$ & $0.62 \pm 0.45$ & 0.021 \\
\hline Fibrosis zone 3 & $0.28 \pm 0.21$ & $0.29 \pm 0.32$ & $0.34 \pm 0.44$ & $0.37 \pm 0.49$ & 0.091 \\
\hline p (z 1 vs. z3) & 0.48 & 0.14 & 0.11 & 0.042 & \\
\hline
\end{tabular}


Table 4. Histological features of NAFLD associated with (hyperuricemia, (uric acid $>5.9$ $\mathrm{mg} / \mathrm{dl})$

\begin{tabular}{|c|c|c|c|}
\hline & $\begin{array}{l}\text { Uric acid } \\
<5.9 \mathrm{mg} / \mathrm{dl}\end{array}$ & $\begin{array}{l}\text { Uric acid } \\
\geq 5.9 \mathrm{mg} / \mathrm{dl}\end{array}$ & $P$ value* \\
\hline Steatosis zone 1 & 1.06 & 1.03 & 0.450 \\
\hline Steatosis zone 3 & 0.96 & 1.01 & 0.550 \\
\hline$p$ (z 1 vs. z3) & 0.87 & 0.33 & \\
\hline Inflammation zone 1 & 0.57 & 0.99 & 0.043 \\
\hline Inflammation zone 3 & 0.37 & 0.48 & 0.260 \\
\hline$p(\mathrm{z} 1$ vs. z3) & 0.12 & 0.02 & \\
\hline Ballooning zone 1 & 0.19 & 0.23 & 0.790 \\
\hline Ballooning zone 3 & 0.12 & 0.21 & 0.340 \\
\hline$p(\mathrm{z} 1$ vs. z3) & 0.17 & 0.22 & \\
\hline Fibrosis zone 1 & 0.51 & 0.78 & 0.046 \\
\hline Fibrosis zone 3 & 0.25 & 0.24 & 0.864 \\
\hline$p(\mathrm{z} 1$ vs. z3) & 0.04 & 0.02 & \\
\hline
\end{tabular}


Table 5: Associations between both (A) high dietary fructose consumption and (B) hyperuricaemia, and histological parameters in zone 1 and in zone 3

\begin{tabular}{|c|c|c|c|c|}
\hline & \multicolumn{2}{|l|}{ Zone 1 (periportal) } & \multicolumn{2}{|l|}{ Zone 3 (perivenous) } \\
\hline \multicolumn{5}{|c|}{$\begin{array}{l}\text { (A) High dietary fructose consumption } \\
\left(>50^{\text {th }} \text { percentile of dietary fructose intake) }\right.\end{array}$} \\
\hline & Odds ratios (95\% CIs) & $\mathbf{p}$ & Odds ratios (95\% CIs) & $\mathbf{p}$ \\
\hline Steatosis & $1.56(1.12,2.49)$ & 0.02 & $1.21(1.09,2.73)$ & 0.039 \\
\hline Inflammation & $4.29(2.31,5.88)$ & 0.0001 & $3.69(2.14,4.56)$ & 0.001 \\
\hline Ballooning & $1.13(0.91,2.99)$ & 0.73 & $0.79(0.53,1.11)$ & 0.62 \\
\hline Fibrosis & $2.72(1.43,3.76)$ & 0.002 & $1.96(1.24,2.37)$ & 0.003 \\
\hline \multicolumn{5}{|c|}{$\begin{array}{l}\text { (B) Hyperuricemia } \\
\text { (Uric acid } \geq 5.9 \mathrm{mg} / \mathrm{dl} \text { ) }\end{array}$} \\
\hline & Odds ratios (95\% CIs) & $\mathbf{p}$ & Odds ratios (95\% CIs) & $\mathbf{p}$ \\
\hline Steatosis & $2.98(1.65,3.23)$ & $<0.0001$ & $1.14(1.05,1.99)$ & 0.045 \\
\hline Inflammation & $1.71(1.17,2.35)$ & 0.01 & $0.86(0.71,1.04)$ & 0.124 \\
\hline Ballooning & $1.05(0.71,2.16)$ & 0.51 & $0.86(0.53,1.71)$ & 0.671 \\
\hline Fibrosis & $2.65(1.35,2.99)$ & 0.031 & $1.31(1.13,2.17)$ & 0.042 \\
\hline
\end{tabular}

Models adjusted for:

High dietary fructose consumption

Age, BMI, HOMA-IR, Triglycerides and UA adjusted

Hyperuricaemia

Age, BMI, HOMA-IR, Triglycerides and dietary fructose consumption 
Supplementary Table 1: Univariate associations between both uric acid concentration and fructose consumption with histological parameters for zone 1 and 3.

\begin{tabular}{|l|l|l|l|l|}
\hline \multicolumn{2}{|l|}{ Uric Acid (mg/dL) } & Fructose (g/day) \\
\hline Zone 1- periportal \\
\hline & r & $\mathbf{p}$ & r & P \\
\hline Steatosis & 0.59 & $\mathbf{0 . 0 0 1}$ & 0.74 & $\mathbf{0 . 0 0 5}$ \\
\hline Inflammation & 0.62 & $\mathbf{0 . 0 2 4}$ & 0.82 & $\mathbf{0 . 0 0 0 4}$ \\
\hline Balloning & 0.29 & 0.196 & 0.31 & 0.324 \\
\hline Fibrosis & 0.54 & $\mathbf{0 . 0 3 2}$ & 0.67 & $\mathbf{0 . 0 0 2}$ \\
\hline Zone 3 - perivenous & \multicolumn{3}{l|}{} \\
\hline & $\mathbf{r}$ & $\mathbf{p}$ & $\mathbf{r}$ & $\mathbf{P}$ \\
\hline Steatosis & 0.38 & 0.078 & 0.58 & $\mathbf{0 . 0 3 4}$ \\
\hline Inflammation & 0.23 & 0.415 & 0.21 & 0.435 \\
\hline Balloning & 0.12 & 0.765 & 0.11 & 0.720 \\
\hline Fibrosis & 0.41 & 0.092 & 0.49 & $\mathbf{0 . 0 4 3}$ \\
\hline
\end{tabular}

